Relations industrielles

Industrial Relations

\title{
Gilles Laflamme et coll. (dir.), La flexibilité des marchés du travail au Canada et aux États-Unis
}

\section{Diane-Gabrielle Tremblay}

Volume 46, numéro 1, 1991

URI : https://id.erudit.org/iderudit/050656ar

DOI : https://doi.org/10.7202/050656ar

Aller au sommaire du numéro

Éditeur(s)

Département des relations industrielles de l'Université Laval

ISSN

0034-379X (imprimé)

1703-8138 (numérique)

Découvrir la revue

Citer ce compte rendu

Tremblay, D.-G. (1991). Compte rendu de [Gilles Laflamme et coll. (dir.), $L a$ flexibilité des marchés du travail au Canada et aux États-Unis]. Relations industrielles / Industrial Relations, 46(1), 229-231.

https://doi.org/10.7202/050656ar

Tous droits réservés @ C Département des relations industrielles de l'Université Laval, 1991
Ce document est protégé par la loi sur le droit d'auteur. L'utilisation des services d'Érudit (y compris la reproduction) est assujettie à sa politique d'utilisation que vous pouvez consulter en ligne.

https://apropos.erudit.org/fr/usagers/politique-dutilisation/ 
La flexibilité des marchés du travail au Canada et aux États-Unis, sous la direction de G. Laflamme et al., Genève, Institut international d'études sociaies, 1989, 347 p., ISBN 92-9014-454-8.

La multiplication des statuts d'emploi, travail temporaire, à temps partiel, à contrat, etc. témoigne bien de la flexibilité du marché du travail au Québec et au Canada, comme dans l'ensemble de l'Amérique du Nord. C'est vers la fin des années 1970 qu'a commencé à se manifester cette recherche de flexibilité de la part des entreprises et c'est depuis le début des années 1980 environ que les chercheurs ont commencé à s'intéresser au phénomène. En fait, c'est surtout en Europe que le débat a émergé, les travaux de plusieurs économistes du travail (Michon, Caire, etc.) et de certains macro-économistes (Robert Boyer en particulier) ayant consacré beaucoup d'attention aux diverses manifestations du phénomène dans les différents pays d'Europe. L'ouvrage issu du colloque tenu par le département de relations industrielles de l'Université Laval à l'automne 1988 vient combler un certain vide du côté des analyses québécoises et nord-américaines de ce thème. En effet, outre les travaux d'Américains comme Michael Piore et un numéro de la revue Interventions économiques consacré au thème de la flexibilité du travail et de l'emploi, les chercheurs québécois et canadiens ont très peu analysé le phénomène en termes de «flexibilité». Sans doute est-ce en grande partie dû au peu de contraintes ou de rigidité auxquelles les entreprises nord-américaines sont effectivement soumises du point de vue de la réglementation ou du droit du travail, en particulier en ce qui concerne les formes d'emploi. De plus, le phénomène des «concessions» salariales et autres a bien montré la flexibilité des marchés du travail en Amérique du Nord.

Le thème de ce livre est donc tout à fait pertinent puisque les contributions qu'il regroupe nous permettent de préciser ce que sous-tend le concept de flexibilité au Québec, au Canada et en Amérique du Nord, par opposition aux sens qu'il recouvre dans divers pays européens. Les articles de Gilles Laflamme et de Lise Poulin-Simon décrivent ainsi la réalité nord-américaine, alors que celui de Guy Standing permet de repérer des oppositions avec l'Europe, tout en fournissant une excellente revue de l'effritement du consensus d'après-guerre, donc une bonne explication des causes du développement des formes d'emploi différenciées et de la flexibilité des marchés du travail. L'article de Standing est par ailleurs un des rares, avec celui de Gerry Rodgers, portant également sur l'Europe, à fournir quelques données chiffrées sur les réalités décrites. C'est d'ailleurs là la principale critique que l'on peut faire de l'ouvrage: le manque de données statistiques permettant d'étayer les analyses présentées. L'analyse du phénomène de la flexibilité est relativement bien couverte dans nombre d'articles, celui de Laflamme présentant notamment des distinctions pertinentes entre la flexibilité salariale, numérique et fonctionnelle, bien que d'autres terminologies soient plus souvent employées: flexibilité des coûts de main-d'oeuvre, de l'emploi (statut et nombre) et flexibilité techno-organisationnelle. De toute évidence, le marché du travail québécois, comme les marchés canadien et américain sont caractérisés par certaines formes de flexibilité plus que d'autres et il eût été intéressant de trouver un article montrant clairement les différences entre l'Amérique du Nord et l'Europe, avec données à l'appui.

Au Québec, les entreprises bénéficient surtout d'une flexibilité que l'on peut associer aux formes d'emploi précaires, et en particulier aux contrats à durée déterminée ou au travail à temps partiel, auquel on peut parfois ajouter des heures de travail au besoin. Cette forme de flexibilité résulte essentiellement des années qui ont suivi les récessions de 1974-1975 et du début des années 1980; nombre d'employeurs ont alors cherché à retrouver la rentabilité perdue au cours des années de récession. Pour d'autres, il s'agissait tout simplement de profiter d'une conjoncture économique défavorable pour obtenir des concessions (salariales ou autres) de la part des salariés. Cela ressort clairement d'un certain nombre de textes qui retracent des situations propres à des entreprises données (General Motors du Canada, Air Canada), mais 
également de textes qui font état des politiques gouvernementales face à la précarité ou à la flexibilité (ceux de Diane Bellemare et de Anthony Giles), ainsi que des stratégies ou attitudes syndicales (Gregor Murray, David Robertson). Dans les années de crise, syndicats et salariés étaient mieux disposés à l'égard de remises en question de certaines conditions de travail et échelles salariales, et les craintes ou défis associés à la conclusion d'une entente de libreéchange entre le Canada et les États-Unis font renaître le débat sur les différentes formes de flexibilité et les alternatives à la flexibilité du travail, dont les coûts reposent essentiellement sur les salariés. Un certain nombre de textes font ainsi écho au contexte du libre-échange et à ses conséquences du point de vue de la flexibilité du travail. Les textes signés par Richard Belous, Gérard Docquier, Judith Maxwell et Claude Rioux donnent différentes perspectives sur la question.

Il est évident que la flexibilité ne plaît généralement pas aux syndicats, qui sont les premiers à s'opposer aux différentes formes de «flexibilité» qui se traduisent par des pertes de statut ou de salaires. En fait, tout en cherchant à protéger les conditions «normales» d'emploi des anciens syndiqués, les syndicats se sont cependant parfois trouvés à encourager la précarisation de l'emploi des nouveaux entrants sur le marché du travail, ou à accepter des échelles salariales inférieures pour les plus jeunes. Plusieurs syndicats ont été lents à accepter d'intégrer et de défendre comme membres à part entière les travailleurs à temps partiel, occasionnels ou temporaires. Il eût peut-être été intéressant de procéder à une analyse plus systématique de ces évolutions et des rôles respectifs de syndicats et des gouvernements dans la différenciation des formes d'emploi et la flexibilité des marchés du travail. Un certain nombre de textes retracent par contre le rôle des employeurs dans l'évolution et les formes prises par la flexibilité. Ainsi, le texte de Michael Piore présente-t-il une excellente revue des stratégies patronales américaines, alors que celui de Jacques Bélanger fait état d'une stratégie axée sur la flexibilité technoorganisationnelle, tout comme celui de R.R. Curd dans une certaine mesure.

Ces textes ont l'immense avantage de mettre en relief le rôle déterminant des gestionnaires dans le choix de l'une ou l'autre des formes de flexibilité et en particulier d'illustrer des stratégies où la flexibilité est recherchée davantage du côté de l'innovation, de l'organisation du travail et des nouvelles technologies plutôt que du côté de l'effritement des statuts d'emploi et des salaires. La flexibilité technico-organisationnelle ou fonctionnelle (cf. Laflamme) est liée à l'articulation du travail et de la technologie de production. Ce dernier aspect de la flexibilité renvoie essentiellement à la polyvalence des équipements productifs, mais également à la polyvalence des travailleurs. En effet, les groupes autonomes, les équipes polyvalentes et autres organisations du genre peuvent fournir ce type de flexibilité à l'entreprise puisqu'elles facilitent l'adaptation à des produits ou des méthodes de production nouvelles. Ces stratégies semblent plus fréquentes dans des pays comme l'Allemagne ou la Suède, mais il est certes intéressant et important de mettre en lumière les (trop rares) expériences du genre menées en Amérique.

Au Québec, il y a eu peu de réflexion sur les diverses options possibles (par ex. variation de la durée d'utilisation des équipements et aménagement du temps de travail, comme le propose Dominique Taddei en France; cf. Le temps de l'emploi, publié chez Hachette). On a plutôt cherché à «flexibiliser» ou à rendre plus malléable ce qui apparaissait à première vue trop rigide, en particulier les statuts d'emploi, les heures «normales» de travail et les avantages sociaux associés à l'emploi régulier à plein temps.

Par opposition, en Suède ou en Allemagne, la formation professionnelle, le recyclage, la mobilité des travailleurs sont mis à profit pour accompagner et même accélérer le changement, de manière à demeurer à la tête du peloton des entreprises ou des nations industrielles. Il faut reconnaître qu'un grand nombre d'institutions facilitent une telle reconversion dans des pays comme l'Allemagne ou la Suède, alors qu'elles sont plutôt absentes du contexte nord- 
américain: des relations de travail plutôt consensuelles, des systèmes de formation, d'apprentissage et de recyclage déjà bien établis notamment. Cela donne au système une grande facilité d'adaptation au changement, sans que les travailleurs n'aient à en payer la note, du moins en termes de précarisation d'emploi. Face à la crise, à l'accentuation de la concurrence internationale, aux changements technologiques accélérés, ces pays ont choisi de faciliter l'adaptation des entreprises et des travailleurs par le biais de leurs politiques publiques et les entreprises elles-mêmes sont généralement très actives en cette matière, qu'elles bénéficient ou non d'aides publiques.

L'article de Paul Osterman retrace pour sa part la perspective américaine à l'égard de la politique de l'emploi, et surtout à l'égard de la formation professionnelle, et fait notamment état de l'intérêt suscité aux États-Unis par le «modèle allemand». Commentant le texte de Paul Osterman, Paul-Martel Roy revendique pour sa part un renouvellement de la politique canadienne en la matière, tout en se montrant peu optimiste.

Quelques articles de l'ouvrage font par ailleurs état de la législation nord-américaine en matière de travail et de flexibilité (Donald Carter, Michael Lynk). Ce dernier présente une bonne comparaison avec les États-Unis, alors que le premier fait une revue assez vaste de l'évolution des lois du travail au Canada. Les deux nous donnent un bon aperçu de l'encadrement juridique de la flexibilité dans le contexte nord-américain; il est simplement à regretter, encore là, que l'on n'ait pas fait un effort plus systématique de comparaison avec l'Europe, afin de bien mettre en relief les éléments qui apportent de la flexibilité par opposition à ceux qui représentent des contraintes dans l'un et l'autre des systèmes de relations industrielles.

Les quelques critiques mentionnées ici n'enlèvent rien à la qualité de la plupart des articles réunis dans cet ouvrage; elles sont peut-être davantage liées aux contraintes et caractéristiques propres des ouvrages regroupant des contributions de divers auteurs réunis au moment d'un colloque. En effet, la grande majorité des textes sont intéressants et instructifs en soi, mais il manque quelques fils conducteurs, quelques liens et éléments de comparaison ou renvois entre les différents articles. Tout de même, étant donné le nombre réduit de publications portant sur le thème de la flexibilité en Amérique du Nord, il faut reconnaître l'intérêt d'une telle publication.

Diane-Gabrielle TREMBLAY

Télé-université, Université du Québec

Économie du travail. Les réalités et les approches théoriques, par Diane-Gabrielle TrEMBLAY, Télé-université et Éditions Saint-Martin, 1990, 544 p., ISBN 2-7624-0171-2 (Téléuniversité) et ISBN 2-89035-155-6 (Éditions Saint-Martin).

Ce livre est présenté comme un manuel d'introduction à l'économie du travail mais, surtout dans sa partie théorique, poursuit des objectifs beaucoup plus ambitieux. Il glisse souvent vers une critique de l'histoire de la pensée économique au détriment d'une exposition claire, étape par étape, des différents modèles explicatifs du marché du travail.

Le livre est structuré en deux grandes parties distinctes. Dans les quatre premiers chapitres, l'auteure fait le pari d'exposer certaines réalités du marché du travail avant d'aborder les approches théoriques. Au total, cette première section couvre plus de 200 pages.

C'est certainement cette section qui correspond le plus à un niveau d'introduction à l'économie du travail. Le ton est un peu journalistique et l'auteure prend le temps de donner 